ARTICLE

\title{
Efficient room temperature catalytic synthesis of alternating conjugated copolymers via C-S bond activation
}

\author{
Zijie Li ${ }^{1,2}$, Qinqin Shi (10) ${ }^{1,2}$, Xiaoying Ma ${ }^{3}$, Yawen $\mathrm{Li}^{4}$, Kaikai Wen ${ }^{1}$, Linqing Qin ${ }^{1}$, Hao Chen ${ }^{1}$, Wei Huang (i) ${ }^{5}$, \\ Fengjiao Zhang ${ }^{3}$, Yuze Lin (10) ${ }^{4}$, Tobin J. Marks (10) ${ }^{5 凶}$ \& Hui Huang (i) $1,2,6,7 凶$
}

Structural defects in conjugated copolymers are severely detrimental to the optoelectronic properties and the performance of the resulting electronic devices fabricated from them. Therefore, the much-desired precision synthesis of conjugated copolymers with highly regular repeat units is important, but presents a significant challenge to synthetic materials chemists. To this end, aryl sulfides are naturally abundant substances and offer unrealized potential in cross-coupling reactions. Here we report an efficient room temperature polycondensation protocol which implements aryl disulfide C-S activation to produce defectminimized semiconducting conjugated copolymers with broad scope and applicability. Thus, a broad series of arylstannanes and thioethers are employed via the present protocol to afford copolymers with number-average molecular weights $\left(M_{n} \mathrm{~s}\right)$ of 10.0-45.0 kDa. MALDI and NMR analysis of selected copolymers reveals minimal structural defects. Moreover, the polymer trap density here is smaller and the field effect mobility higher than that in the analogous polymer synthesized through thermal-activation Stille coupling.

\footnotetext{
${ }^{1}$ College of Materials Science and Opto-Electronic Technology, University of Chinese Academy of Sciences, Beijing 100049, P. R. China. ${ }^{2}$ Center of Materials Science and Optoelectronics Engineering, University of Chinese Academy of Sciences, Beijing 100049, P. R. China. ${ }^{3}$ School of Chemical Sciences, University of Chinese Academy of Sciences, Beijing 100049, P. R. China. ${ }^{4}$ Beijing National Laboratory for Molecular Sciences, CAS Key Laboratory of Organic Solids, Institute of Chemistry, Chinese Academy of Sciences, Beijing 100190, P. R. China. ${ }^{5}$ Department of Chemistry and the Materials Research Center,

Northwestern University, 2145 Sheridan Road, Evanston, IL 60208, USA. ${ }^{6}$ CAS Center for Excellence in Topological Quantum Computation, University of Chinese Academy of Sciences, Beijing 100049, P. R. China. ${ }^{7}$ CAS Key Laboratory of Vacuum Physic, University of Chinese Academy of Sciences, Beijing

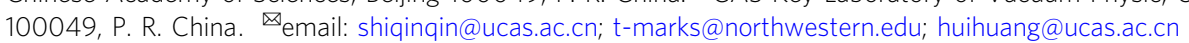


S emiconducting polymers, especially $\pi$-conjugated donoracceptor (D-A) copolymers with defect-free alternating structures, are essential materials for plastic electronics and optoelectronics owing to their attractions of variable/tunable chemical structures, tunable bandgaps and optical absorptions, excellent charge transport mobility, mechanical flexibility, and solution processability for printing ${ }^{1-3}$. However, to synthesize such complex macromolecules, thermally activated cross-couplings, such as Suzuki-Miyaura, Stille, direct arylation polymerization (DArP), etc., have become the standard procedure ${ }^{4-6}$. Nevertheless, the high reaction temperatures of these polymerizations not only consume energy, but can also compromise chemoselectivity by producing inseparable structural defects in the product polymers ${ }^{7-10}$. Up until now, the polymerizations that can be performed at room temperature or lower in short reaction times have primarily utilized methodologies such as controlled/ living polymerization or radical polymerization ${ }^{11,12}$. However, the major limitation of these approaches is lack of regioselectivity, hence unsuitability for creating device-quality alternating conjugated polymers ${ }^{13}$.

Recently, the significant need for atom-efficient and environmentally benign chemical synthesis has increased, and turned to process energy-efficiency and starting materials and reagents that must conform to these requirements ${ }^{14}$. Organosulfur compounds that are widely available from nature ${ }^{15-17}$ often serve as effective electrophiles in transition metal-assisted cross-coupling reactions, such as Liebeskind-Srogl (L-S) coupling in the case of small molecules (Fig. 1a) ${ }^{18}$. Thus, abundant aromatic thioethers offer a sustainable and environmentally friendlier alternative to traditional aryl halide reagents ${ }^{19}$. Furthermore, C-S bonds are well tolerated functional groups in most other catalytic crosscoupling reactions, such as Suzuki, Stille, etc. ${ }^{20,21}$, and importantly, the potentially competing homocoupling of C-S bonds has never been reported, implying that inert C-S bonds will avoid or minimize homocoupling by-products ${ }^{22-24}$. Finally, C-S bond activation using unique additives may substantially lower the activation energies of the desired coupling/polymerization reactions ${ }^{22,24}$, thereby allowing polymerization reactions to proceed near room temperature. Therefore, developing a universal and efficient catalytic system to realize the cross-coupling polymerization of aryl thioethers or aryl stannanes based on C-S bond activation presents an intriguing opportunity.

Here we report on the scope and mechanism of a room temperature step-wise cross-coupling polymerization process utilizing aromatic thioethers as electrophiles, representing, to our knowledge, the unprecedent cross-coupling polymerization based on C-S activation (Fig. 1b). Competition experiments indicate that transmetalation is the rate-determining step, indicating that the coupling reaction occurs rapidly between electron-donating nucleophiles and aromatic thioethers, affording more rapid polymerization rates and higher molecular masses than conventional thermal Stille couplings. A wide substrate scope of electrondonating and electron-withdrawing substituted nucleophiles demonstrates the generality of this methodology. Moreover, the present defect studies on the polymers synthesized by our method demonstrate relatively clean NMR spectra, small trap densities, and high hole mobilities, in comparison to those obtained by thermally-activated Stille coupling polymerization.

\section{Results and discussion}

Polymerization optimization and scope. Monomer BBT-2SMe (Table 1, E1), a derivative of natural waste 2-(methylthio)-benzothiazole $(\mathrm{MTBT})^{25}$, synthesized using a lithiation-mediated sulfide transfer reaction, was used as the principal test electrophile in the following polycondensations with 2,6-bis(trimethyltin)-4,8bis(2-octyldodecyloxy)-benzo[1,2-b:3,4-b)]dithiophene selected as the nucleophile (Table 1; Supplementary Table 1). Since elevated temperatures ensure solubility of the growing polymer chain, an initial polymerization between E1 and N1 using $\mathrm{Pd}\left(\mathrm{PPh}_{3}\right)_{4}$ as the catalyst and copper(I) thiophene-2carboxylate $(\mathrm{CuTc})$ as the cocatalyst was performed at $100^{\circ} \mathrm{C}$ (Table 1 entry 1 ) to afford P1 with $M_{\mathrm{n}}=14.5 \mathrm{kDa}$ and $Ð$ (polydispersity index) $=4.82$. When the reaction temperature was lowered to $60^{\circ} \mathrm{C}$, the product polymer had a similar molecular mass and polydispersity $\left(M_{\mathrm{n}}=14.3 \mathrm{kDa}, Ð=3.39\right)$ (entry 2). Impressively, a similar product in similar yield is obtained at $25^{\circ} \mathrm{C}\left(M_{\mathrm{n}}=15.7 \mathrm{kDa}, Ð=3.15\right)$ which is unprecedented for Stille-type polymerizations (entry 3 ). Not surprisingly, the polymerization affords a lower molecular mass product $\left(M_{\mathrm{n}}=7.4\right.$ $\mathrm{kDa}$ ) and yield when the reaction temperature is lowered to $0{ }^{\circ} \mathrm{C}$. The reaction time is also an important factor in energy efficiency, and was screened from 12 (entry 5) to $72 \mathrm{~h}$ (entry 6). Both reactions afford polymers with $M_{\mathrm{n}}, Ð$, and yield similar to those in $24 \mathrm{~h}$. Thus, $12-24 \mathrm{~h}$ may be a suitable time range for polymer chain growth. During the polymerization in the above entries, an immediate color change from colorless to deep-red indicates a rapid reaction early stage, consistent with a step-wise mechanism ${ }^{26}$. The amount of catalyst/cocatalyst is also critical for reaction efficiency. Thus, lowering either the Pd (entry 7) or $\mathrm{Cu}$ (entry 8) loading results in a dramatic fall in product $M_{\mathrm{n}}$ (but not yield). Next the types of $\mathrm{Cu}$ cocatalyst were also screened (entries 9-10), which revealed that a copper carboxylate is optimal, in contrast to previous reports on L-S reactions ${ }^{24,27}$.

Since the specific identity of the catalyst is the key factor for these polycondensations, other Pd catalysts were screened (Supplementary Table 1, entries 1-2) for further optimization, which showed that $\mathrm{Pd}\left(\mathrm{PPh}_{3}\right)_{4}$ is the most efficient. In this

a Classic Stille Cross-Coupling Polymerization via C-X activation

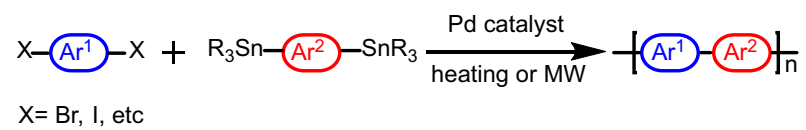

b This Work: Cross-Coupling Polymerization via C-S activation

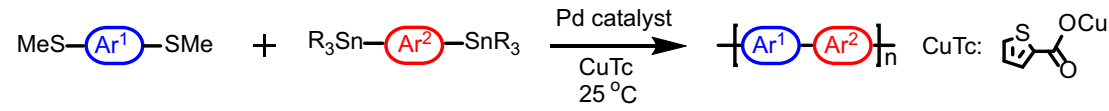

Advantages: energy- and atom-efficient; homocoupling defects minimized.

Fig. 1 Synthesis of $\boldsymbol{\pi}$-conjugated donor-acceptor copolymers. a Classic Stille cross-coupling polycondensation. $\mathbf{b}$ The present cross-coupling polymerization via L-S reaction (Liebeskind-Srogl reaction). 
Table 1 Optimization of C-S cleavage polycondensation conditions for the synthesis of semiconducting polymer P1 from E1 + N1a.

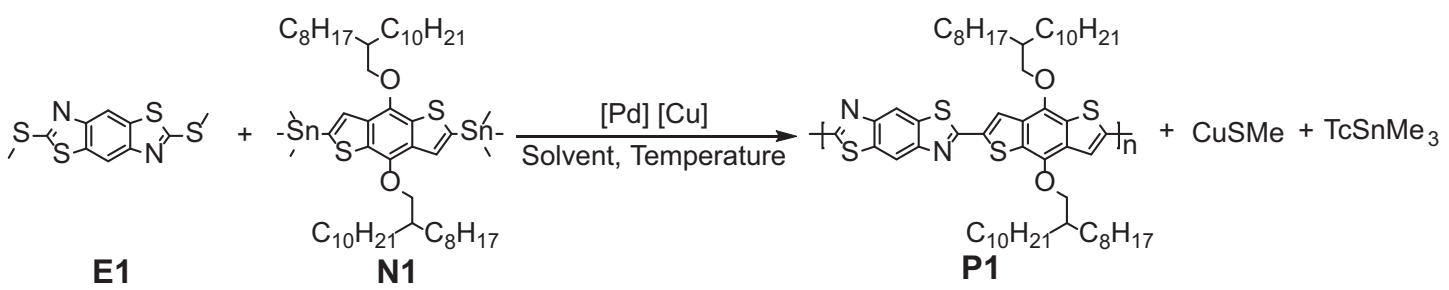

\begin{tabular}{|c|c|c|c|c|c|c|c|c|}
\hline Entry & [Pd] Catalyst & [Cu] Cocatalyst & Solvent & $\mathrm{T}\left({ }^{\circ} \mathrm{C}\right)$ & Time (h) & $M_{\mathrm{n}} / M_{\mathrm{w}}(\mathrm{kDa})$ & Ð & Yieldb (\%) \\
\hline 1 & $\mathrm{Pd}\left(\mathrm{PPh}_{3}\right)_{4}$ & CuTc & Toluene & 100 & 24 & $14.5 / 70.0$ & 4.82 & 84 \\
\hline 2 & $\mathrm{Pd}\left(\mathrm{PPh}_{3}\right)_{4}$ & CuTc & Toluene & 60 & 24 & $14.3 / 48.7$ & 3.39 & 81 \\
\hline 3 & $\mathrm{Pd}\left(\mathrm{PPh}_{3}\right)_{4}$ & CuTc & Toluene & RT & 24 & $15.7 / 49.5$ & 3.15 & 84 \\
\hline 4 & $\mathrm{Pd}\left(\mathrm{PPh}_{3}\right)_{4}$ & CuTc & Toluene & 0 & 24 & $7.4 / 23.4$ & 3.17 & 24 \\
\hline 5 & $\mathrm{Pd}\left(\mathrm{PPh}_{3}\right)_{4}$ & CuTc & Toluene & RT & 12 & $15.1 / 73.3$ & 4.85 & 60 \\
\hline 6 & $\mathrm{Pd}\left(\mathrm{PPh}_{3}\right)_{4}$ & CuTc & Toluene & RT & 72 & $15.9 / 60.5$ & 3.81 & 70 \\
\hline $7^{c}$ & $\mathrm{Pd}\left(\mathrm{PPh}_{3}\right)_{4}$ & CuTc & Toluene & RT & 24 & $4.7 / 12.2$ & 2.62 & $62^{c}$ \\
\hline $8^{d}$ & $\mathrm{Pd}\left(\mathrm{PPh}_{3}\right)_{4}$ & CuTc & Toluene & RT & 24 & $3.7 / 7.9$ & 2.10 & $80^{d}$ \\
\hline 9 & $\mathrm{Pd}\left(\mathrm{PPh}_{3}\right)_{4}$ & CuMeSal & Toluene & RT & 24 & $7.8 / 20.9$ & 2.67 & 52 \\
\hline 10 & $\mathrm{Pd}\left(\mathrm{PPh}_{3}\right)_{4}$ & Cul & Toluene & RT & 24 & - & - & 0 \\
\hline
\end{tabular}

Reaction conditions: apd catalyst (10 mol\%), Cu cocatalyst (5 equiv.), E1 (1 equiv, $0.025 \mathrm{M}$ ) and $\mathbf{N 1}$ (1 equiv, $0.025 \mathrm{M}$ ) in solvent under $\mathrm{N}_{2}$

bYield collected from chloroform fraction.

CPd $\left(\mathrm{PPh}_{3}\right)_{4}(5 \mathrm{~mol} \%)$; yield collected from hexane fraction

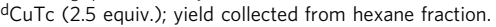

Table 2 Polycondensation of P1 in classic Stille coupling between E2 and N1a.

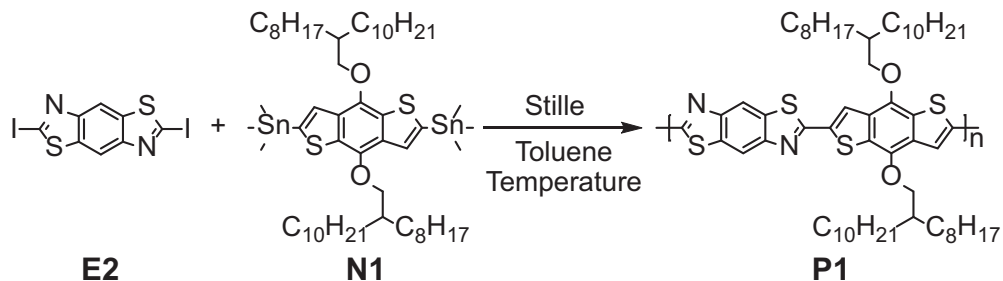

\begin{tabular}{llllll}
\hline Entry & Catalyst & Temperature & $\mathbf{M}_{\mathbf{n}} / \mathbf{M}_{\mathbf{w}} \mathbf{( k D a )}$ & $\boldsymbol{D}$ & Yieldb(\%) \\
\hline 1 & $\mathrm{Pd}\left(\mathrm{PPh}_{3}\right)_{4}$ & - & - & 0 \\
2 & $\mathrm{Pd}\left(\mathrm{PPh}_{3}\right)_{4}$ & $100^{\circ} \mathrm{C}$ & $11.1 / 46.1$ & 4.17 & 82 \\
$3^{\mathrm{C}}$ & $\mathrm{Pd}\left(\mathrm{PPh}_{3}\right)_{4}, \mathrm{CuTc}$ & $100^{\circ} \mathrm{C}$ & $7.5 / 22.6$ & 3.04 & 61 \\
\hline
\end{tabular}

Reaction conditions: aPd catalyst ( $10 \mathrm{~mol} \%$ ), E1 (1 equiv, $0.025 \mathrm{M})$ and $\mathbf{N 1}\left(1\right.$ equiv, $0.025 \mathrm{M}$ ) in solvent under $\mathrm{N}_{2}$ at room temperature (entry 1 ) or $100{ }^{\circ} \mathrm{C}$ (entry 2 )

bYield collected from chloroform fraction.

${ }^{\mathrm{C} C u T c}$ (5 equiv.), $100^{\circ} \mathrm{C}$.

polymerization process, the solvent is expected to affect both the catalyst stability and product molecular mass ${ }^{28,29}$. The reaction solvent chlorobenzene (CB) affords polymers with a slightly higher $M_{\mathrm{n}}$ values than toluene (entry 3), which may be ascribed to the better product solubility in the former. Next the more environmentally acceptable solvents, THF, dioxane, and DMF were also screened (entries 4-6), however the polymerization efficiency falls dramatically in all three solvents. Finally, reaction solution concentration effects were scrutinized. Fine tuning the monomer concentrations to 0.1 and $0.001 \mathrm{M}$ (Supplementary Table 1, entries 7-8) yields polymers with $M_{\mathrm{n}}$ below $10 \mathrm{kDa}$, suggesting the baseline $0.025 \mathrm{M}$ conditions are near optimal in terms of efficiency and molecular mass (Table 1, entry 3). To verify batch-to-batch reproducibility, four parallel trials were performed under the optimized conditions. The product P1 exhibits nearly identical $M_{\mathrm{n}}$ over $15.0 \mathrm{kDa}$ and comparable yields over $80 \%$ (Supplementary Table 2). Additionally, a 1.0 gram scale reaction affords P1 with $M_{\mathrm{n}} / M_{\mathrm{w}}$ of $15.2 / 58.6 \mathrm{kDa}$ and a yield of
$85 \%$ in chloroform fraction (Supplementary Table 2). Therefore, this protocol is quite robust, excellent reproducibility and scalability without obvious fluctuation in $M_{\mathrm{n}}$ and yield.

To provide a direct comparison between classic Stille and the present C-S activation based cross-coupling polymerizations, three parallel reactions were carried out in coupling 2,6diiodobenzo[1,2-d:4,5-d'] bis(thiazole) (E2) and N1 under various conditions (Table 2 ). The reaction of entry 1 maintains a colorless solution over 3 days, suggesting negligible activity in a classic Stille polymerization at $25^{\circ} \mathrm{C}$. On increasing the temperature to $100^{\circ} \mathrm{C}$, the reaction affords a polymer with a $M_{\mathrm{n}}=11.1 \mathrm{kDa}$ and $Ð=4.17$ after 3 days (Table 2 entry 2), arguing that classic high temperature Stille is less efficient than the $25^{\circ} \mathrm{C} \mathrm{C}-\mathrm{S}$ activation based polycondensation. Interestingly, addition of the $\mathrm{CuTc}$ cocatalyst to the Stille system gives inferior activity versus the classic Stille methodology (Table 2 entry 3). Therefore, present copolymerization approach via C-S activation is superior to classic Stille cross-coupling in this system. Moreover, the polymer 
a Competition Reactions

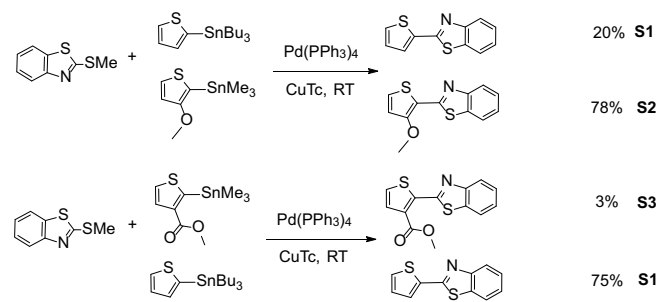

b Control Experiments

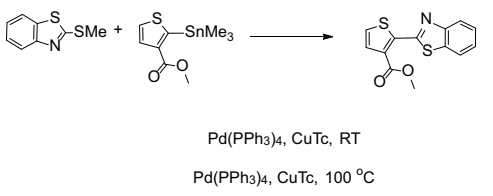

$\mathrm{Pd}\left(\mathrm{PPh}_{3}\right)_{4}, \mathrm{CuTc}, \mathrm{Zn}(\mathrm{OAc}) 2, \mathrm{RT}$ c Proposed Mechanism

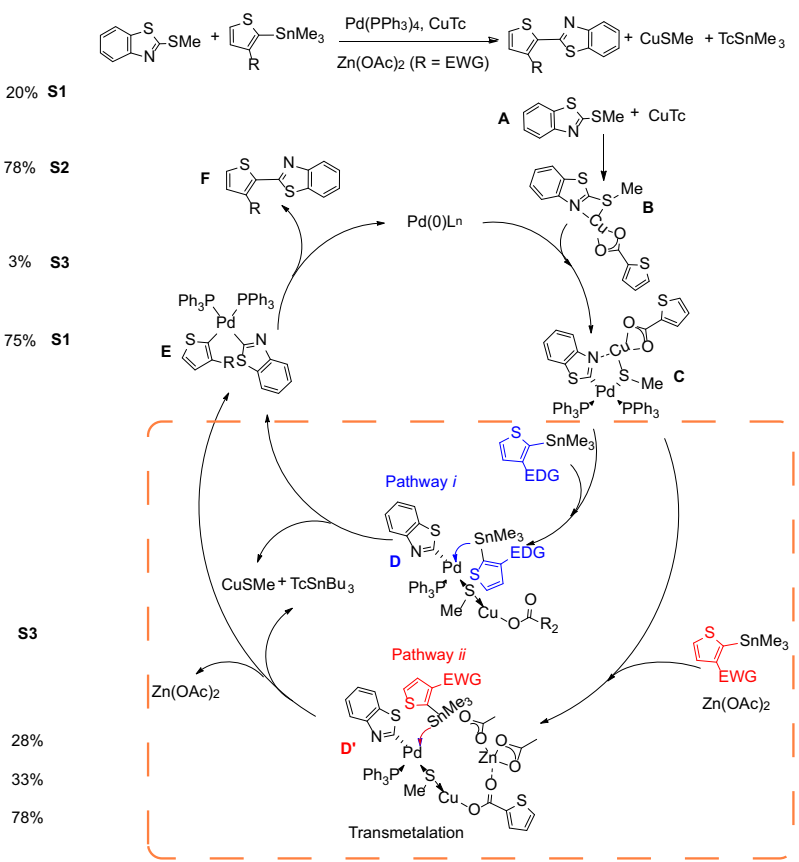

Fig. 2 Mechanism studies. a Competition reactions and b Control experiment studies of aryl stannane activity and selectivity. c Plausible mechanism for C-S cleavage-based cross-coupling polymerization. All yields were determined by gas chromatography (GC) analysis using anthracene as internal standard.

${ }^{1} \mathrm{H}$ NMR of the high temperature Stille and the present $25^{\circ} \mathrm{C} \mathrm{C}-\mathrm{S}$ activation products are indistinguishable, with comparable line widths (Supplementary Fig. 1). Additionally, the MALDI-TOF of P1 (Supplementary Fig. 2) exhibits the expected repeat units corresponding to C-S/C-Sn cross-coupling products $(m / z=970)$, and the end group analysis is consistent with the presence of both monothioether/H and dithioether termini.

Model reactions and mechanistic considerations. Small molecule reactions can aid in understanding the utility of a synthetic polymerization method. Note that classic Stille coupling proceeds via a step-growth polymerization mechanism ${ }^{26}$, and in this scenario high-yielding reactions are essential for producing high $M_{\mathrm{w}}$ polymers, which is critical to the regularity and electronic properties of the resulting conjugated polymers ${ }^{30}$. To probe the electronic effects of the aryl stannane, competition reactions were performed (Fig. 2a). The direct competition reactions between MTBT, 2-(trimethylstannyl)thiophene, and 2-(trimethylstannyl) thiophene-3-methoxy affords 2-(thiophen-2-yl)benzo[d]thiazole (S1) and 2-(3-methoxythiophen-2-yl)benzo[d] thiazole (S2) with GC yields of $20 \%$ and $78 \%$, respectively. In contrast, competition between MTBT, 2-(trimethylstannyl)thiophene, and 2-(trimethylstannyl)thiophene-3-carboxylate led to GC yields of $75 \%$ and $3 \%$ for $\mathbf{S 1}$ and methyl-2-(benzo[ $d]$ thiazol-2-yl)thiophene-3carboxylate (S3), respectively. These results argue that the more electron-rich stannane nucleophile is more reactive, and that transmetalation is reasonably the rate-determining step in crosscoupling. To investigate the influence of the electron-poor nucleophiles, control experiments in the model reaction between 2-(trimethylstannyl)thiophene-3-carboxylate and MTBT were next conducted. The results reveal that only by heating is the yield increased to 33\% (Fig. 2b). Interestingly, addition of $\mathrm{Zn}(\mathrm{OAc})_{2}$ dramatically enhances the yield to $c a$. $80 \%$, consistent with literature reports on related reactions ${ }^{31}$. Based on above studies, a tentative mechanism (Fig. 2c) is proposed, highlighting the importance of the transmetalation step. It was hypothesized that the $\mathrm{C}-\mathrm{S}$ bond was activated by $\mathrm{CuTc}$ which then facilitates the subsequent $\mathrm{Pd}(0)$ oxidative addition ${ }^{18}$. The resultant $\mathrm{Pd}(\mathrm{II})$ complex (C) undergoes a transmetalation with electron-rich 2(trimethylstannyl)thiophene in Pathway $i$. For the transmetalation with an electron-poor 2-(trimethylstannyl)thiophene-3-carboxylate, $\mathrm{Zn}(\mathrm{OAc})_{2}$ plays a critical role in assisting transmetalation in Pathway ii. Finally, both pathways involve reductive elimination to afford the target diaryls.

Reaction scope. The generality of this catalytic protocol was examined by investigating the coupling of electrophiles E1-E4 with a series of nucleophiles (N2-N9) to afford the corresponding conjugated copolymers P2-P11. Table 3 summarizes all polymerizations under the optimized reaction conditions. As electronrich and rigid ladder-type moieties ${ }^{32,33}$, IDT and IDTy distannanes (N2 and N3) were copolymerized with E1 to smoothly afford copolymers P2 and P3 in good yields, respectively. Specifically, $\mathbf{P} 2$ achieves a moderate $M_{\mathrm{n}}$ of $13.4 \mathrm{kDa}$ in a high yield of $82 \%$, mainly due to the strong electron-donating ability of $\mathrm{N} 2$. Meanwhile, reflecting the introduction of branched alkyl chains, the molecular weight of P3 is further increased, affording a $22 \%$ yield fraction in CB $(34.7 \mathrm{kDa})$. Copolymer $\mathbf{P 4}$ was reported to have the highest hole mobility for a BBT-based polymer, when prepared by classic Stille coupling with a $M_{\mathrm{n}}$ of $26 \mathrm{kDa}^{34}$. In the present work, $\mathbf{P 4}$ was prepared via cross-coupling of E1 and N4, yielding a higher $M_{\mathrm{n}}$ of $30.5 \mathrm{kDa}$ in $60 \%$ yield. Note, however, that this reaction yields $c a .30 \%$ insoluble residue, implying that polymer chain growth is constrained by solubility. Next, the sidechain dimensions of the terthiophenes were increased to $n$ octyldodecyl. Not surprisingly, copolymer P5 achieves an overall yield of $83 \%$ with $M_{\mathrm{n}}=45.0 \mathrm{kDa}$. Furthermore, two more electron-rich units N6 and N7 were copolymerized with E1 to afford polymers $\mathbf{P 6}$ and P7, respectively, with moderate $M_{\mathrm{n}}$ s and good yields. Since electron-withdrawing moieties are poor nucleophiles in this protocol, nucleophilic DPP distannanes (N8) 
Table 3 General applicability of C-S cleavage protocol to other $\pi$-electron polymers.

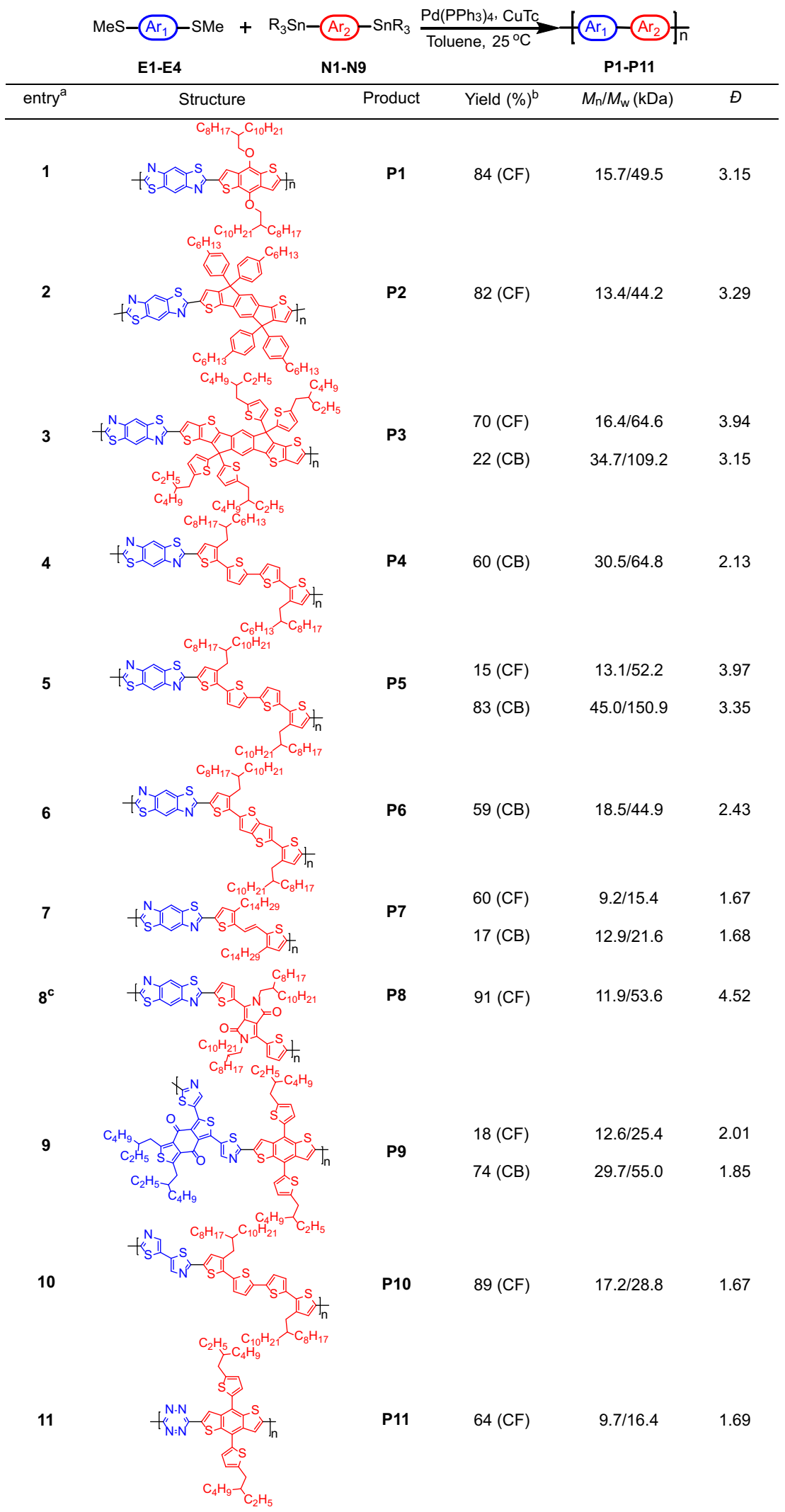

Reaction conditions: aPd $\left(\mathrm{PPh}_{3}\right)_{4}(10 \mathrm{~mol} \%)$, CuTc (5 equiv.), monomers in toluene $(0.025 \mathrm{M})$ for $24 \mathrm{~h}$ under $\mathrm{N}_{2}$

${ }^{C P d}\left(\mathrm{PPh}_{3}\right)_{4}(10 \mathrm{~mol} \%)$, CuTc (5 equiv.), $\mathrm{Zn}(\mathrm{OAc})_{2}(10 \mathrm{~mol} \%)$, monomers in toluene $(0.025 \mathrm{M})$ for $72 \mathrm{~h}$ under $\mathrm{N}_{2}$. 
could not successfully generate polymer P8 in 3 days under the above optimal conditions. Building on Scheme $2 \mathrm{~b}, \mathrm{Zn}(\mathrm{OAc})_{2}$ was next used as an additive in this polymerization. Copolymer $\mathbf{P 8}$ is obtained with $M_{\mathrm{n}} / M_{\mathrm{w}}=11.9 / 53.6 \mathrm{kDa}$ in $91 \%$ yield. Next, arene thioethers E2, E3, and $\mathbf{E 4}$ were screened in reactions with nucleophiles N9 and N5 to afford P9, P10, and P11 with $M_{\mathrm{n}}$ s ranging from 29.7 to $9.7 \mathrm{kDa}$, respectively, in good yields, suggesting broad generality of electrophilic thioethers. Finally, 2,5bis(methylsulfanyl)thiophene and 1,4-bis(methylsulfanyl)benzene were screened as electrophiles. However, no polymers were obtained in the reactions, which may be ascribed to the absence of $\mathrm{N}$ atom in the electrophiles that can coordinate with $\mathrm{CuTc}$ to facilitate the C-S bond activation. The polymer structures were confirmed with ${ }^{1} \mathrm{H}$ NMR, high temperature GPC, and elemental analysis. In all, this synthetic methodology is effective for both electrophiles and electron-rich and electron-deficient nucleophiles, suggesting broad future applications.

The photophysical properties of the present P1-P11 polymers in $\mathrm{CHCl}_{3}$ solution and as thin films were investigated by UV-vis and photoluminescence spectroscopy. The corresponding spectra are shown in Supplementary Figs. 4 and 5, respectively, and the data are summarized in Supplementary Table 3. All polymers in solution exhibit a single major absorption band, which corresponds to intramolecular charge transfer (ICT) excitations at $300-750 \mathrm{~nm}$ in solution. The corresponding thin film absorptions for polymers P1-P3, P7-P8, and P11 exhibit slight redshifts due to modest $\pi-\pi$ stacking $^{35}$. In comparison, P4-P6 and P9-P10 thin films show large redshifts of over $21 \mathrm{~nm}$ versus their solution spectra, indicating strong interchain $\pi-\pi$ stacking in the solid state. The individual photoluminescence spectra of all polymers are shown in Supplementary Fig. 5. P1-P7 and P9-P11 solutions in $\mathrm{CHCl}_{3}$ fluoresce with quantum yields of $1.4,35.6,26.4,14.8$, $7.2,5.0,1.3,1.0,6.2$, and 1.5\%, respectively, with Stokes shifts ranging from 2 to $115 \mathrm{~nm}$. Interestingly, $\mathbf{P 8}$ exhibits no emission, which may be ascribed to strong donor - acceptor unit ${ }^{35}$. Cyclic voltammetry $(\mathrm{CV})$ with the ferrocene/ferrocenium $\left(\mathrm{Fc} / \mathrm{Fc}^{+}\right)$ redox couple as an external standard was carried out to characterize the redox properties of all polymers (Supplementary Fig. 6; Supplementary Table 3). The lowest unoccupied molecular orbital (LUMO) and highest occupied molecular orbital (HOMO) energy levels of P1-P11 are estimated to lie at $-2.87 /-5.42$, $-2.80 /-5.44,-2.96 /-5.58,-2.81 /-5.37,-2.73 /-5.28,-2.94 /$ $-5.50,-2.99 /-5.13,-3.62 /-5.25,-3.50 /-5.55,-2.80 /-5.37$, and $-3.56 /-5.68 \mathrm{eV}$, respectively. The relatively high-lying HOMO levels of P1-P7 and P9-P11 suggest potential p-type semiconductors, while the low-lying LUMO of P8 suggest possible n-type electron transporting properties (see more below). The decomposition temperatures characterized by thermogravimetric analysis (TGA) of P1-P11 are all above $300^{\circ} \mathrm{C}$ (Supplementary Fig. 7 and Supplementary Table 3), suggesting excellent thermal stability.

Defect studies. Homocoupling between electrophiles or nucleophiles are a major deleterious side reaction of cross-coupling reactions, which may cause structural defects in the polymer backbone ${ }^{8}$. To investigate the importance of such side reactions in cross-coupling via L-S type reactions and classic Stille reactions, four parallel experiments were performed (Fig. 3a). Interestingly, in the L-S type reaction, trace amounts of homocoupling product S5 between nucleophiles but no homocoupling product S4 between electrophiles are observed in model reactions at $100{ }^{\circ} \mathrm{C}$, while homocoupling products S5 and S4 were not observed at room temperature by GC analysis. In comparison, the yield of homocoupling product S4 is significant $(27 \%)$ at $100^{\circ} \mathrm{C}$ in the classic Stille coupling between 2-(tributylstannyl)thiophene and 2-iodobenzo[d]thiazole. Not surprisingly, a significant amount of homocoupling product of electrophile S4 (4\%) is still observed for the classic Stille coupling at room temperature. This direct comparison argues that cross-coupling via the present C-S cleavage is plausibly a superior methodology to suppress homocoupling defects in alternating conjugated polymer via catalytic synthesis.

Since P2 (also refer to P2-CS) exhibited sharp peaks in aromatic region of ${ }^{1} \mathrm{H}$ NMR which is facile to identify the potential homocoupling structural defects. Next, the resulting polymers P2-CS (also refer to P2) and P2-CI were synthesized by our room temperature C-S cleavage method and classic thermal Stille coupling, respectively, to have a direct comparison on structural defects, trap density and further organic field effect transistors (OFET) applications. The MALDI-TOF of P2-LS (Supplementary Fig. 3) exhibit the expected repeat units corresponding to C-S/C-Sn cross-coupling products $(m / z=1094)$ indicating the alternating pattern of the polymer backbone. Afterwards, by comparing the aromatic

region of the ${ }^{1} \mathrm{H}$ NMR spectra of P2-CS and P2-CI (Figs. 3b, c), the integration of additional proton peak $\boldsymbol{a}$ ' for P2-CI are more than that of P2-CS, which may be from the potential homocoupling defects. To further identify the defects ratio of P2CS and P2-CI, PBBT and PIDT were synthesized via thermal Stille coupling. After the careful assignment of PBBT and PIDT (Supplementary Fig. 3, Figs. 3b, c) chemical shifts, proton $\boldsymbol{a}$ ' and $\boldsymbol{a}$ " of PBBT can be assigned at 8.5 and $7.9 \mathrm{ppm}$ which could be applied to identify the BBT homocoupling (hc) ratios of P2-CS and P2-CI. Basically, the content of BBT homocoupling junctions of P2-CS is found to be less than 7\%, consistent with the results of the model reactions. In comparison, the homocoupling defects are more than 19\% in P2-CI (Fig. 3c). This direct comparison underscores the attraction of lower temperature cross-coupling polymerization via C-S cleavage. Although several studies argue that the charge transport mobilities of copolymer is robust against homocoupling structural defects in the OFETs, a deep understanding and interpretation from the trap densities perspective is necessary ${ }^{36,37}$. Here, thermal admittance spectroscopy (TAS) analysis $^{38}$ was used to quantize the reduction of trap states in P2-CS and P2-CI films, respectively. As shown in Fig. 3d, the energetic profile of trap density of states (tDOS) demonstrated a density of defects states on the order of $10^{17}$ to $10^{19}$ for P2-LS film, which is relatively smaller than that of P2-CI for both shallow and deep defects in the films. Next, OFETs were fabricated with polymers P2-CS and P2-CI for comparison of the charge transport. As shown in Fig. 3e, f, Supplementary Fig 9, and Supplementary Table 4, the P2 based OFETs exhibit a good hole mobility of $0.12 \mathrm{~cm}^{2} /$ Vs with a high $I_{\text {on/off }}$ (on-to-off current ratio) of $c a .10^{5}$. While, P2-CI OFETs have a lower hole mobility of $0.06 \mathrm{~cm}^{2} / \mathrm{Vs}$ with $I_{\text {on/off }}$ of $c a .10^{6}$, which may reflect the less ordered crystallinity (Supplementary Fig. 10-12) and larger density defects. Again, the results argue that $\mathrm{C}-\mathrm{S}$ activation based cross-coupling polymerization at room temperature can produce conjugated polymers with comparable or superior microstructural and electronic properties to those from classic Stille coupling at high temperature.

In addition, nanoparticles of P2-LS copolymer exhibit excellent co-localization coefficients of 0.79 with lysosome, highlighting the potential application of BBT-based polymers for cell imaging (Supplementary Fig. 13-17). Next, polymer P4 and P5 exhibit excellent field-effect transistor hole mobilities (Supplementary Fig. 18-20), rivaling or exceeding literature metrics for the same or comparable materials, further demonstrating the high quality of resulting polymers ${ }^{34}$ (Detail discussions are in Supplementary Information).

In summary, we have established a broadly applicable room temperature aryl disulfide C-S polycondensation methodology for 


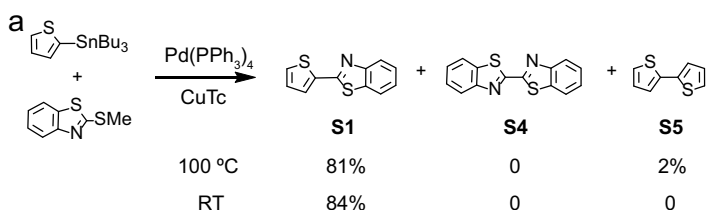

b

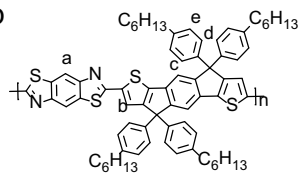

P2-CS: $M_{\mathrm{n}} / M_{\mathrm{w}}=13.4 / 44.2 \mathrm{kDa}$

P2-Cl: $M_{\mathrm{n}} / M_{\mathrm{w}}=10.2 / 15.2 \mathrm{kDa}$

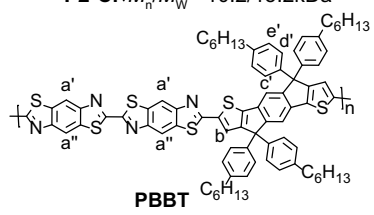

C

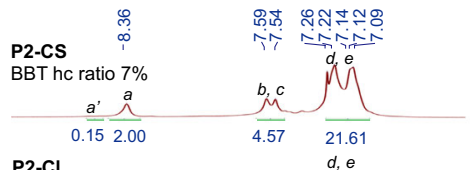

P2-Cl

BBT hc ratio $19 \%$

$\underline{a_{i}} \stackrel{a}{\Lambda}$

$\frac{a_{u}^{\prime}}{0.47} \frac{\lambda}{2.00}$

b,c
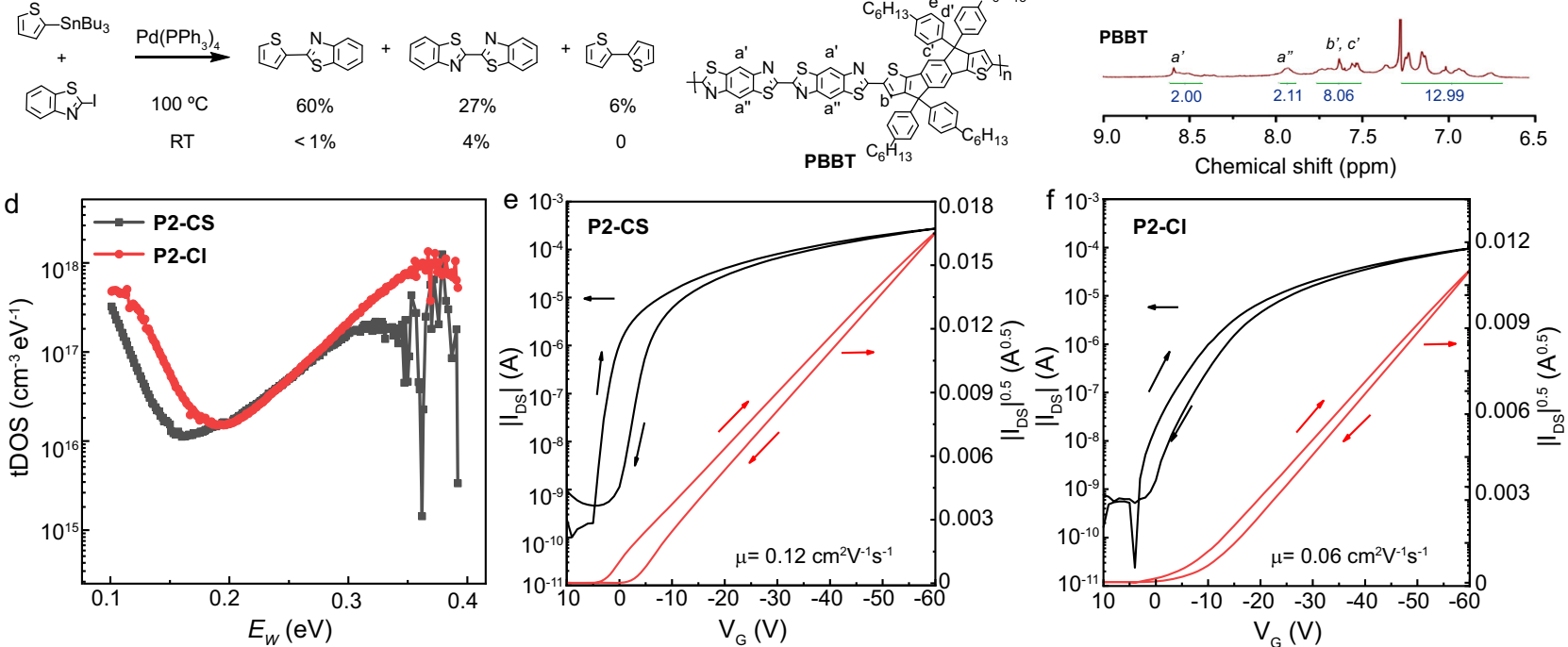

Fig. 3 Defects studies. a Side reaction study on generation of structural defects. All yields were determined by gas chromatography (GC) analysis using anthracene as internal standard; $\mathbf{b}$ The structures of P2-CS, P2-CI, and PBBT synthesized by room temperature Stille via C-S cleavage, thermal Stille via C-I cleavage and thermal Stille, respectively; c The aromatic region of ${ }^{1} \mathrm{H}$ NMR for $\mathbf{P 2}-\mathbf{C S}, \mathbf{P 2}-\mathbf{C l}$, and $\mathbf{P B B T}$ from 9.0 to $6.5 \mathrm{ppm}$; $\mathbf{d}$ Trap density of states (tDOS) obtained by thermal admittance spectroscopy of P2-CS and P2-CI thin films; e OFET transfer curves of polymer P2-CS thin film; $\mathbf{f}$ OFET transfer curves of polymer $\mathbf{P 2}-\mathbf{C l}$ thin film.

the efficient cross-coupling polymerization between diverse aryl distannanes and aryl bis-thioethers to produce defect-minimized, high molecular mass alternating semiconducting conjugated copolymers. In comparison to polymer counterparts synthesized via classic Stille polycondensation, the present protocol produces higher molecular masses and defect-reduced polymers in room temperature polymerizations. Detailed mechanistic studies with competition reactions, control experiments, and side reaction analyses provide further guidelines for optimizing on polymer synthesis and minimizing defect generation. A series of alternating polymers was synthesized and shown to have suitable energy levels for semiconduction. It is noteworthy that this structural defect increases the defect density in the bulk films which results in a low OFET hole mobility. Therefore, this polycondensation will not only provide a robust and competitive method for polymer synthesis but also help minimizing the structure defects in high performance polymer semiconductors.

\section{Methods}

Procedure for polymerizations. Into a Schlenk flask, E1-E4 (1 equiv), N1-N9 (1 equiv), $\mathrm{CuTc}$ (5 equiv), $\mathrm{Pd}\left(\mathrm{PPh}_{3}\right)_{4}(10 \mathrm{~mol} \%)$ and dry toluene $(0.025 \mathrm{M})$ were added under a nitrogen atmosphere. The reaction mixture was then stirred at room temperature for $24 \mathrm{~h}$, or with $10 \mathrm{~mol} \%$ of $\mathrm{Zn}(\mathrm{OAc})_{2}$ as an additive for 3 days. Afterwards, the reaction mixture was poured into methanol. The crude polymer was collected by filtration and purified by successive Soxhlet extractions with acetone, hexane, chloroform and chlorobenzene. The chloroform (CF) or chlorobenzene (CB) solution was then poured into methanol and collected by filtration to afford corresponding product copolymers.

OFET fabrication and characterization. A heavily doped Si wafer with a $300 \mathrm{~nm}$ thermally grown $\mathrm{SiO}_{2}$ layer served as gate and dielectric layer, respectively. Before the deposition of the polymers (P2-CS and P2-CI), the substrates were treated with octadecyltrichlorosilane (OTS) in a vacuum oven at a temperature of $120^{\circ} \mathrm{C}$, forming an OTS self-assembled monolayer. After rinsing by hexane, ethanol and chloroform, $10 \mathrm{mg} / \mathrm{mL}$ polymer solution in chlorobenzene was spun coated with a speed of $5000 \mathrm{rpm}$, subsequently annealed at $180^{\circ} \mathrm{C}$ for $60 \mathrm{~min}$. On top of the polymer thin films, Au source-drain electrodes $(30 \mathrm{~nm})$ were thermally evaporated, where the channel length and channel width are $50 \mu \mathrm{m}$ and $5000 \mu \mathrm{m}$, respectively. The OFETs measurements were carried out in ambient using Keysight B2900A analyzer. The mobility in saturation region was calculated with the equation, $\mu=\frac{2 L}{W C_{o x}}\left(\frac{d \sqrt{I_{S D}}}{d V_{G}}\right)^{2}$, where $\mathrm{I}_{\mathrm{SD}}$ is the source-drain current, $\mathrm{V}_{\mathrm{G}}$ corresponds to gate voltage, $\mathrm{L}$ and $\mathrm{W}$ represent the channel length and channel width, respectively, $\mathrm{C}_{\mathrm{ox}}$ means the capacitance the $\mathrm{SiO}_{2}$ layer which equals to $11 \mathrm{nF} / \mathrm{cm}^{2}$.

\section{Data availability}

The authors declare that the all the data supporting the finding of this study are available within this article and its Supplementary Information files and are available from the corresponding author on reasonable request.

Received: 31 August 2021; Accepted: 10 December 2021; Published online: 10 January 2022

\section{References}

1. Heeger, A. J. Semiconducting polymers: the third generation. Chem. Soc. Rev. 39, 2354-2371 (2010).

2. Qiu, Z., Hammer, B. A. G. \& Müllen, K. Conjugated polymers-problems and promises. Prog. Polym. Sci. 100, 101179 (2020).

3. Pouliot, J.-R., Grenier, F., Blaskovits, J. T., Beaupré, S. \& Leclerc, M. Direct (hetero)arylation polymerization: simplicity for conjugated polymer synthesis. Chem. Rev. 116, 14225-14274 (2016).

4. Osedach, T. P., Andrew, T. L. \& Bulović, V. Effect of synthetic accessibility on the commercial viability of organic photovoltaics. Energy Environ. Sci. 6, 711-718 (2013)

5. Dudnik, A. S. et al. Tin-free direct C-H arylation polymerization for high photovoltaic efficiency conjugated copolymers. J. Am. Chem. Soc. 138, 15699-15709 (2017)

6. $\mathrm{Ni}$, Z. et al. Mesopolymer synthesis by ligand-modulated direct arylation polycondensation towards n-type and ambipolar conjugated systems. Nat. Chem. 11, 271-277 (2019). 
7. Stephens, C. \& Sweat, D. Synthesis and Stille cross-coupling reactions of 2(tributylstannyl)- and 2,5-bis(trimethylstannyl)tellurophene. Synthesis 2009, 3214-3218 (2009).

8. Rudenko, A. E. \& Thompson, B. C. Optimization of direct arylation polymerization (DArP) through the identification and control of defects in polymer structure. J. Polym. Sci. Part A: Polym. Chem. 53, 135-147 (2015).

9. Zhang, X. et al. Synthesis and characterization of monodisperse oligo(fluorene-co-bithiophene)s. Chem. - A Eur. J. 13, 6238-6248 (2007).

10. Vittorio, F. New perspectives in the cross-coupling reactions of organostannanes. Pure Appl. Chem. 68, 73-78 (1996).

11. Zhang, H. H., Xing, C. H. \& Hu, Q. S. Controlled $\mathrm{Pd}(0) /{ }^{t-}-\mathrm{Bu}_{3} \mathrm{P}$-catalyzed Suzuki cross-coupling polymerization of $\mathrm{AB}$-type monomers with $\mathrm{PhPd}\left(\mathrm{t}^{\mathrm{t}}\right.$ $\left.\mathrm{Bu}_{3} \mathrm{P}\right) \mathrm{I}$ or $\mathrm{Pd}_{2}(\mathrm{dba})_{3} /{ }^{t-} \mathrm{Bu}_{3} \mathrm{P} / \mathrm{ArI}$ as the initiator. J. Am. Chem. Soc. 134, 13156-13159 (2012).

12. Mayhugh, A. L. \& Luscombe, C. K. Room-temperature Pd/Ag direct arylation enabled by a radical pathway. Beilstein J. Org. Chem. 16, 384-390 (2020).

13. Yokozawa, T. \& Ohta, Y. Transformation of step-growth polymerization into living chain-growth polymerization. Chem. Rev. 116, 1950-1968 (2016).

14. Phan, S. \& Luscombe, C. K. Recent advances in the green, sustainable synthesis of semiconducting polymers. Trends Chem. 1, 670-681 (2019).

15. Drushel, H. V. \& Miller, J. F. Polarographic estimation of thiophenes and aromatic sulfides in petroleum. Anal. Chem. 30, 1271-1280 (1958).

16. Nawrocki, S. T., Drake, K. D., Watson, C. F., Foster, G. D. \& Maier, K. J. Comparative aquatic toxicity evaluation of 2-(thiocyanomethylthio) benzothiazole and selected degradation products using Ceriodaphnia dubia. Arch. Environ. Contamination Toxicol. 48, 344-350 (2005).

17. Zhou, X. \& Chriswell, C. Determination of forms of organic sulfur in coal by step-wise oxidation with perchloric acid-ferric perchlorate mixture. Fuel Process. Technol. 46, 41-47 (1996).

18. Liebeskind, L. S. \& Srogl, J. Thiol ester-boronic acid coupling. A mechanistically unprecedented and gene-ral ketone synthesis. J. Am. Chem. Soc. 122, 11260-11261 (2000).

19. Pan, F. \& Shi, Z.-J. Recent advances in transition-metal-catalyzed C-S activation: from thioester to (hetero)aryl thioether. ACS Catal. 4, 280-288 (2014).

20. Krömer, M., Klečka, M., Slavětínská, L., Klepetářová, B. \& Hocek, M. Chemoselective synthesis of 4,5-diarylpyrrolo[2,3-d]pyrimidines (6,7-diaryl-7deazapurines) by consecutive suzuki and Liebeskind-Srogl cross-couplings. Eur. J. Org. Chem. 2014, 7203-7210 (2014).

21. Prieur, V., Pujol, M. D. \& Guillaumet, G. A strategy for the triarylation of pyrrolopyrimidines by using microwave-promoted cross-coupling reactions. Eur. J. Org. Chem. 2015, 6547-6556 (2015).

22. Lou, J. et al. Transition-metal mediated carbon-sulfur bond activation and transformations: an update. Chem. Soc. Rev. 49, 4307-4359 (2020).

23. Wang, L., He, W. \& Yu, Z. Transition-metal mediated carbon-sulfur bond activation and transformations. Chem. Soc. Rev. 42, 599-621 (2013).

24. Cheng, H.-G., Chen, H., Liu, Y. \& Zhou, Q. The Liebeskind-Srogl crosscoupling reaction and its synthetic applications. Asian J. Org. Chem. 7, 490-508 (2018).

25. Zhu, T. et al. Converting thioether waste into organic semiconductors by carbon-sulfur bond activation. Angew. Chem. Int. Ed. 58, 5044-5048 (2019).

26. Flory, P. J. Fundamental principles of condensation polymerization. Chem. Rev. 39, 137-197 (1946).

27. Li, H., Yang, H. \& Liebeskind, L. S. Synthesis of high enantiopurity $\mathrm{N}$-protected $\alpha$-amino ketones by thiol ester-organostannane cross-coupling using pH-neutral conditions. Org. Lett. 10, 4375-4378 (2008).

28. Bao, Z., Chan, W. K. \& Yu, L. Exploration of the Stille coupling reaction for the synthesis of functional polymers. J. Am. Chem. Soc. 117, 12426-12435 (1995).

29. Lee, S. M., Park, K. H., Jung, S., Park, H. \& Yang, C. Stepwise heating in Stille polycondensation toward no batch-to-batch variations in polymer solar cell performance. Nat. Commun. 9, 1867 (2018).

30. Shi, Q. et al. The direct arylation polymerization (DArP) of well-defined alternating copolymers based on 5,6-dicyano[2,1,3]benzothiadiazole (DCBT). Asian J. Org. Chem. 7, 1419-1425 (2018).

31. Liebeskind, L. S. \& Srogl, J. Heteroaromatic thioether-boronic acid crosscoupling under neutral reaction conditions. Org. Lett. 4, 979-981 (2002).

32. Yan, C. et al. Non-fullerene acceptors for organic solar cells. Nat. Rev. Mater. 3, 18003 (2018).
33. Cheng, P., Li, G., Zhan, X. \& Yang, Y. Next-generation organic photovoltaics based on non-fullerene acceptors. Nat. Photonics 12, 131-142 (2018).

34. Osaka, I., Takimiya, K. \& McCullough, R. D. Benzobisthiazole-based semiconducting copolymers showing excellent environmental stability in high-humidity air. Adv. Mater. 22, 4993-4997 (2010).

35. Noriega, R. et al. A general relationship between disorder, aggregation and charge transport in conjugated polymers. Nat. Mater. 12, 1038-1044 (2013).

36. Wang, Q. et al. Electron mobility of diketopyrrolopyrrole copolymers is robust against homocoupling defects. Chem. Mater. 33, 668-677 (2021).

37. Hong, W. et al. Is a polymer semiconductor having a "perfect" regular structure desirable for organic thin film transistors? Chem. Sci. 6, 3225-3235 (2015).

38. Shao, Y. et al. Origin and elimination of photocurrent hysteresis by fullerene passivation in $\mathrm{CH}_{3} \mathrm{NH}_{3} \mathrm{PbI}_{3}$ planar heterojunction solar cells. Nat. Commun. 5, 5784-5491 (2014).

\section{Acknowledgements}

The authors acknowledge the financial support from the NSFC (21774130, 51925306, and 21905277), National Key R\&D Program of China (2018FYA 0305800), Key Research Program of the Chinese Academy of Sciences (XDPB08-2), the Strategic Priority Research Program of Chinese Academy of Sciences (XDB28000000), and Fundamental Research Funds for the Central University. T.J.M. thanks the National Science Foundation Materials Research Science and Engineering Center at Northwestern University (DMR-1720319) for support.

\section{Author contributions}

H.H. conceived the work. Z.L. and Q.S. performed the synthetic experiments and characterization and contributed equally to this work. Y. Li and Y. Lin contributed to trap density measurement and analysis. L.Q., X.M. and F.Z. performed the device fabrication and analysis. K.W. performed the cell imaging. H.C. performed the photoluminescence test. W.H. performed the GIWAXS test. Z.L., Q.S., T.J.M., and H.H. wrote the manuscript. H.H. provided overall supervision.

\section{Competing interests}

The authors declare no competing interests.

\section{Additional information}

Supplementary information The online version contains supplementary material available at https://doi.org/10.1038/s41467-021-27832-1.

Correspondence and requests for materials should be addressed to Qinqin Shi, Tobin J. Marks or Hui Huang.

Peer review information Nature Communications thanks Anne Staubitz and the other, anonymous, reviewer(s) for their contribution to the peer review of this work. Peer reviewer reports are available.

\section{Reprints and permission information is available at http://www.nature.com/reprints}

Publisher's note Springer Nature remains neutral with regard to jurisdictional claims in published maps and institutional affiliations.

Open Access This article is licensed under a Creative Commons Attribution 4.0 International License, which permits use, sharing, adaptation, distribution and reproduction in any medium or format, as long as you give appropriate credit to the original author(s) and the source, provide a link to the Creative Commons license, and indicate if changes were made. The images or other third party material in this article are included in the article's Creative Commons license, unless indicated otherwise in a credit line to the material. If material is not included in the article's Creative Commons license and your intended use is not permitted by statutory regulation or exceeds the permitted use, you will need to obtain permission directly from the copyright holder. To view a copy of this license, visit http://creativecommons.org/ licenses/by/4.0/.

(c) The Author(s) 2022 